\title{
La lectura entre sociología y massmediología
}

\author{
Elena Ranfa
}

Artículo recibido:

9 de noviembre de 2015

Artículo aceptado:

29 de septiembre de 2016

\section{Resumen}

La complejidad de la lectura y de los muchos pasos que han caracterizado la historia de la lectura ha estimulado, desde el siglo pasado, una serie de reflexiones bajo diferentes perspectivas. En el artículo trataremos de centrar la atención en una de estas contribuciones, es decir, el resultado de dos disciplinas fuertemente conectadas, como la sociología y la massmediología. En particular, se considerarán los argumentos y las obras de los académicos pertenecientes a la denominada Escuela de Toronto, un grupo heterogéneo de intelectuales que en la segunda mitad del siglo pasado empezó con el estudio de la relación entre la evolución de las

* Università degli Studi di Perugia, Italia elena.ranfa@gmail.com

INVESTIGACIÓN BIBLIOTECOLÓGICA, vol.32, núm.75, abril/junio, 2018, México, ISSN: 2448-8321 pp. 83-108 
tecnologías del habla y los cambios cognitivos y socioculturales. Los puntos iniciales de estos autores se pueden resumir en una aproximación al estudio de los medios interdisciplinarios, pero especialmente en una clara tendencia a considerar la tecnología, sobre todo la tecnología de las comunicaciones, como una variable independiente en el estudio de los procesos de cambios sociales.

Palabras clave: Lectura; Medios de comunicación; Sociología; Escuela de Toronto

\section{Reading from sociology to mass media studies Elena Ranfa}

\section{Abstract}

The intricacies of reading and its manifold developments throughout history have inspired numerous studies conducted from diverse perspectives. This paper attempts to highlight the unique contributions of sociology and mass communication research, specifically addressing the theories and works produced by the members of the Toronto School, a diverse group of scholars, who during the second half of the twentieth century began to explore the connections between emerging technologies of speech, and those associated with cognitive, cultural and social changes. The starting points of these inquiries can be summarized through an approach to the study of interdisciplinary media, especially communications technology as an independent variable in the study of social change.

Keywords: Reading; Media; Sociology; Toronto School 


\section{INTRODUCCIÓN}

En una cultura como la nuestra, con una larga tradición de fraccionar y dividir para controlar, puede ser un choque que le recuerden a uno que, operativa y prácticamente, el medio es el mensaje. Esto significa simplemente que las consecuencias individuales y sociales de cualquier medio, es decir, de cualquiera de nuestras extensiones, resultan de la nueva escala que introduce en nuestros asuntos cualquier extensión o tecnología nueva.

Marshall McLuhan

E 1 artículo ahonda sus raíces en un proyecto de investigación en el cual se ha analizado la figura del "lector híbrido", es decir, aquel lector que está viviendo importantes momentos de transición, de paso, en los cuales prácticas y herramientas que parecían consolidadas y tal vez inmutables sufren transformaciones profundas, auténticas "revoluciones" que condicionan, de manera más o menos consciente, su vida. Entendiendo con el término híbrido no sólo la coexistencia de elementos diferentes en una misma realidad, sino también la transformación y el crecimiento de una específica realidad a otra, en la cual también los elementos de continuidad se encuentran renovados, se ha intentado delinear la figura del lector a partir de un examen teórico de las aproximaciones de diferentes disciplinas que se han ocupado de manera transversal de los medios de comunicación, del papel del lector, del acto de leer, de las prácticas y de los espacios de la lectura y de todas las posibles declinaciones que un tema tan complejo como el de la lectura suscita. La actividad de leer y los múltiples tránsitos que caracterizaron la historia de la lectura, de hecho, han estimulado, a partir del siglo pasado, una serie de reflexiones y de aproximaciones que parten de diferentes perspectivas.

El punto de vista examinado en esta contribución atañe al ámbito de la sociología y de la massmediología. En particular para el estudio y el análisis de estos momentos de transición, se ha considerado fundamental la profundización de las teorías y las intuiciones de los intelectuales miembros de la llamada Escuela de Toronto.

A estos estudiosos se les ha otorgado el crédito de haber sido los primeros en individuar y analizar, a través de una aproximación científica fuertemente renovada, la estrecha conexión existente entre la evolución de la civilización humana y la de los instrumentos de la comunicación. 
Pese a haber sido a menudo acusada de un excesivo determinismo tecnológico (por leer de forma demasiado rígida y automática la relación entre el cambio social y cultural y los modelos a través de los cuales se expresa la tecnología en las diversas sociedades), a la Escuela de Toronto se le reconoce el mérito no sólo de haber abierto el enfrentamiento sobre temáticas fuertemente actuales, como el rol de los medios de comunicación en la sociedad, sino también de haber ejercido una influencia (no siempre reconocida explícitamente) en filones de estudios que, desde puntos de vista diferentes, han situado en el centro de su reflexión a los media y el impacto que ejercen sobre el hombre.

Los puntos desde los que parten los exponentes de la Escuela se centran en la nueva manera de considerar los medios: la tecnología se ve como el motor del cambio, una fuerza que puede determinar la dirección del cambio de la sociedad. Para los autores, cada tecnología lleva consigo un bias, es decir, una tendencia a la conservación del saber. Todos los medios (desde el papiro hasta las modernas tecnologías) han evolucionado en una especie de escala: el nuevo medio englobaba el anterior y la información en él contenida. Ocurrieron auténticas mutaciones antropológicas, como las del hombre. Los mismos medios se consideran como una especie de extensión del hombre (la escritura, extensión de la memoria; el teléfono, extensión de voz y oído) pero también como una especie de extensión de la conciencia. Tales procesos llevan al nacimiento de la aldea global, con la consiguiente diferenciación entre medios calientes (estimulan un solo sentido, como la radio y el cine) y medios fríos (sobreentienden una mayor participación por parte del usuario, por ejemplo, televisión, teléfono e internet).

La Escuela de Toronto tiende, a modo de síntesis, a desinteresarse por el contenido de los medios, privilegiando el estudio del medio en sí, puesto que el contenido del medio es también otro medio: el contenido de la escritura es el discurso, de la misma manera que la palabra escrita está contenida en la imprenta, y este punto de vista hace de ella un punto de partida interesante para analizar una historia de la lectura que inevitablemente es también historia de los soportes.

\section{Oralidad y lectura. LAS “TRANSFORMACiONES DE LA PALABRA”}

Punto de partida ha sido el encuentro con aquella que está considerada como la obra más importante (o por lo menos más conocida en Italia) del estudioso norteamericano Walter Jackson Ong, Oralità e scrittura. Le tecnologie della 
parola (Oralidad y escritura. Las tecnologías de la palabra), que se sitúa plenamente en el marco de interés que involucró a comienzos de los años 80 a este heterogéneo grupo de intelectuales, es decir, el estudio de las relaciones entre la evolución de las tecnologías de la palabra y los cambios cognitivos y socioculturales. Profunda es, de hecho, la atención que Ong pone en las transformaciones de los mecanismos lingüístico-cognoscitivos en los pasos de la oralidad a la escritura y a la imprenta.

Con Oralità e scrittura, Ong trata de analizar las causas y las modalidades del cambio que se pueden observar en el paso de una cultura prevalentemente oral a una escrita, mirando, sin prejuicios, tanto al pasado lejano como al mundo contemporáneo. Individuando en la escritura (a mano, impresa o electrónica) la clave para leer esta transición, afirmando que ésta haya actuado sobre los cambios de la forma mentis del mundo occidental más de lo que se pueda creer. ${ }^{2}$

El camino empezado por Ong parte del concepto de palabra entendida como principal medio de comunicación a través de la cual el hombre se relaciona con el exterior y como objeto de investigación para volver a recorrer los procesos evolutivos que han caracterizado la historia de la "sociedad humana" (Barilli, 1970): "La relación del hombre con el hombre, del hombre con la sociedad, del hombre con su entero mundo vital, que incluye también su religiosidad, puede ser vista bajo nuevos aspectos estudiando la historia de la palabra misma, es decir, la historia de las comunicaciones..." (Ong, 1970: 22), entendiendo por comunicaciones "no tanto nuevos instrumentos que permitan al hombre 'comunicarse' con sus semejantes, sino de modo más comprehensivo, las maneras que una persona tiene de entrar en la vida y en la personalidad de los otros y, por lo tanto, de su propia vida” (Ong, 1970: 22).

Es la investigación de la palabra en sí sobre la que hay que empezar una reflexión, sólo después de habernos "despojado" de la convicción propia de la civilización de hoy de considerar la palabra como un "documento":

Tendemos a pensar en las palabras como documentos porque tendemos a pensar en ellas como escritas o impresas, considerando que sea este su estado óptimo. Una vez que hayamos conseguido superar este prejuicio quirográfico-tipográfico, podremos ver que la palabra en su babitat sonoro original, el cual es, todavía hoy, su habitat natural, no es en absoluto un documento.

2 En contra de este ajuste que ve en el paso de la oralidad a la escritura una "solución de continuidad entre pensamiento mítico, prelógico y una visión racional del mundo”, toma partido Ludwig Jäger, afirmando que "muchas prestaciones que Ong considera típicas de las culturas literarias ya existen en algunas culturas orales y por lo tanto no son fruto exclusivo del uso de la escritura" (Jäger, 2003: 52). 
La palabra es algo que ocurre, un acontecimiento en el mundo del sonido a través del cual la mente humana es capaz de ponerse en contacto con la realidad. (Ong, 1970:31)

La palabra, según Ong, es antes que nada "hablada" y tiene un vínculo indisoluble, o mejor dicho, se identifica, con el sonido. Sonido que, como los demás sentidos, está ligado al concepto de tiempo y espacio, pero que con respecto al tiempo tiene una relación que el mismo Ong define "especial":

Todas las sensaciones tienen lugar en el tiempo, pero el sonido en concreto tiene una relación especial con el tiempo, distinto de aquel de los otros sectores del sistema sensorial humano. [...] El sonido existe sólo en el momento en el que está muriendo; deteriorable y esencialmente evanescente, y como tal, se percibe. (Ong, 1986: 59)

El sonido se mueve, existe aquí, en este momento; está relacionado con la realidad circunstante y con el presente. El sonido no interfiere con los demás sentidos y, al contrario que la vista, que examina las superficies, el sonido revela un interior a otro interior, sin seccionarlo o descomponerlo.

El sonido y la palabra se sitúan entonces en una posición de superioridad respecto a los otros sentidos y, por lo tanto, respecto a los otros medios a través de los cuales el hombre comunica; de hecho, aunque el hombre comunique con todo su cuerpo, es la palabra su principal medio de comunicación y, por otra parte, la comunicación, como el conocimiento mismo, culmina precisamente en la expresión verbal.

La palabra como tal no tiene entonces una presencia visiva, las palabras "sólo son sonidos que se pueden 'rellamar', recordar, pero no hay ningún lugar en donde 'buscarlos"” (Ong, 1986: 59).

En la cultura oral no existe ningún medio tangible en el cual poder "encontrar" palabras; el único soporte es la memoria del hombre: "En el discurso oral [...] no hay nada a lo que engancharse fuera de la mente, puesto que la expresión oral se desvanece en cuanto se pronuncia" (Ong, 1986: 68).

En un mundo en el que todo lo que se refiere al hombre está encomendado al happening del sonido, en el que la experiencia fluye ininterrumpidamente sin dejarse encerrar, se podrá recordar lo que se ha elaborado sólo recurriendo a precisos módulos mnemónicos:

En una cultura oral primaria, para resolver de manera eficaz el problema de tener en mente o recuperar un pensamiento articulado, es necesario pensar en módulos mnemónicos creados a propósito para una rápida recuperación oral. El pensamiento debe nacer dentro de módulos equilibrados con un 
gran contenido rítmico, debe estructurarse en repeticiones y antítesis, en aliteraciones y asonancias, en epítetos y expresiones formulares, en temas generales, en refranes constantemente oídos por todos y que se recuerdan fácilmente, ellos también formulados para un aprendizaje y recuerdo, o finalmente en otras formas con función mnemónica.

El pensamiento está entrelazado con los sistemas mnemónicos, los cuales determinan también la sintaxis. (Ong, 1989: 237-238)

La sintaxis se somete entonces a las exigencias de la memoria de tal manera que resulte la estructura del lenguaje más agregativa que analítica, más redundante que sintética, más paratáctica que hipotáctica.

En una cultura oral, además, el discurso tiende esencialmente a autoconservarse, minimizando las innovaciones. Incluso reconociéndole una cierta originalidad narrativa que se expresa en la interacción con un público nunca igual a sí mismo y en el tener que enfrentarse en situaciones (políticas, sociales, económicas) siempre diferentes, Ong subraya el carácter marcadamente tradicionalista y conservador de una cultura de oralidad primaria, donde el ejercicio asiduo de la memoria no deja espacio a la experimentación intelectual.

El conocimiento que una cultura de este tipo expresa está extremadamente contextualizado, es el espejo de una sociedad, de un conjunto de individuos que comparten valores, emociones y a los cuales no les interesan en absoluto las definiciones abstractas sino que ponen al día su cultura, y por ende su memoria, en respuesta a las exigencias del momento, del presente.

Es sólo con la escritura que la palabra adquiere una nueva dimensión espacio-temporal, menos huidiza, más duradera: "La escritura encierra la palabra, tiránicamente y para siempre, en un campo visivo" (Ong, 1971: 66). En el paso de una cultura oral a una cultura de lo escrito, alfabetizada, se produce un deslizamiento del ámbito sonoro al visivo; el hombre sufre una verdadera separación física y mental de la palabra (separación que Ong define como "alienación") que se convierte ella misma en una presencia autónoma, concretizándose en letra, frase, periodo escrito.

Para comprender en profundidad el método con el que analiza esta transición y el papel decisivo que le atribuye para leer la historia y los cambios de la civilización y del pensamiento humanos, es necesario detenerse sobre el significado que Ong atribuye al concepto de escritura.

¿Qué es la escritura? La escritura propiamente dicha, o sea, aquella tecnología que ha moldeado y potenciado la actividad intelectual del hombre moderno, fue una invención muy tardía de la historia humana. [...] 
Naturalmente es posible considerar "escritura" cada signo semiótico, es decir, cada signo visible e inteligible producido por un individuo, y al cuál él asigna un significado. De esta manera sería escritura una simple raya sobre una roca o una marca en un palo, interpretable sólo por quien las ha producido. Si esto es lo que se entiende por escritura, su antigüedad es posiblemente comparable con la del discurso oral. Sin embargo las investigaciones que dan esta interpretación del término confunden la escritura con un comportamiento puramente biológico. [...]

Utilizar el término "escritura" en un sentido tan extenso que llega a incluir en él cualquier signo semiótico, lo vacía de significado. La apertura hacia nuevos mundos del conocimiento tuvo lugar en la mente humana, no cuando fue ideado el simple signo semiótico, sino cuando fue inventado un sistema codificado de marcadores visivos a través de los cuales el escribiente podía determinar las palabras exactas que el lector produciría a partir del texto. Esto es lo que nosotros hoy en día entendemos habitualmente por escritura en sentido estricto. [...] La escritura, entendida en este sentido, fue y es el evento de mayor envergadura en la historia de los inventos tecnológicos del hombre. No se trata de un simple apéndice del discurso oral, puesto que transportando el mundo oral-aural a una nueva dimensión del sistema sensorial, la de la vista, la escritura transforma al mismo tiempo discurso y pensamiento. (Ong, 1986: $125-127)$

La escritura es algo artificial, externa al hombre, fuera de su inconsciente, distante de la naturalidad del lenguaje oral y de su carácter marcadamente comunitario. La escritura es una tecnología, una invención del hombre pero no por esto hay que relegarla a un rol subalterno o secundario. Al contrario, cualquier tecnología (sobre todo cuando tiene que ver con la palabra), si adecuadamente interiorizada, no degrada sino que eleva y mejora la vida de la "sociedad humana".

El discurso escrito como tal difiere del discurso oral en el sentido de que no nace en el inconsciente. El transferir la lengua hablada a la escritura es un proceso guiado por normas conscientemente inventadas, y que se pueden formular claramente [...] Decir que la escritura es artificial no significa condenarla, todo lo contrario: al igual y más que cualquier otra creación artificial, ella tiene un valor inestimable, puesto que es esencial para el desarrollo más completo de los potenciales humanos interiores. Las tecnologías no son simples apoyos externos, sino que conllevan transformaciones de las estructuras mentales [...] Tales transformaciones pueden ser positivas: la escritura, por ejemplo, eleva el nivel de conciencia; la alienación de un entorno natural nos puede beneficiar y es de muchas maneras esencial para la plenitud de la vida humana. Para vivir y comprender bien, necesitamos 
no sólo la proximidad, sino también la distancia; esto es lo que la escritura regala a la mente humana de manera única, como ninguna otra cosa puede hacerlo.

Las tecnologías son artificiales, pero - de nuevo la paradoja - la artificialidad es natural para los seres humanos. [...] El uso de una tecnología puede por tanto enriquecer la psique humana, expandir el espíritu, intensificar la vida interior. (Ong, 1986: 123-125)

Alienación y artificialidad son dos conceptos que con la llegada de la imprenta se exacerban aún más; la palabra pasa de ser sonido a letra, a objeto físico: el carácter móvil, matriz de la imprenta. Lo sonoro cede inexorablemente el paso a lo visivo, más de lo que hubiera podido ocurrir con la escritura (pensemos en el lugar en primer plano que la oralidad sigue teniendo en la cultura manuscrita).

La escritura alfabética había subdividido la palabra en los equivalentes espaciales de las unidades fónicas (en teoría, aunque en práctica las letras no ejercieran casi nunca esta función de manera perfecta), pero las letras utilizadas no preexistían al texto en el cual aparecían. Con la imprenta de caracteres móviles las cosas son diferentes: los carácteres tipográficos preexisten a las palabras que tendrán que formar. La imprenta sugiere que las palabras son cosas, más de lo que la escritura hizo nunca. [...] En el mundo del pensamiento y de la expresión, la imprenta sustituyó entonces el prolongado dominio del oído por el de la vista, cuya influencia había empezado con la escritura pero que no habría podido imponerse con su soporte. La imprenta ubica inexorablemente las palabras en el espacio. (Ong, 1986: 170-174)

El camino emprendido por Ong continúa con el análisis de las nuevas transformaciones electrónicas de la expresión verbal, subrayando cómo los nuevos medios de comunicación, en tanto que tecnología, si bien sean aparentemente distantes al hombre, a su inconsciente, expresan en realidad el dinamismo, la riqueza cultural del ser humano. Es el proceso de interiorización que corresponde a cada innovación; medios creados por la genialidad del hombre, que del hombre aparentemente se alejan para volver a entrar otra vez prepotentemente en su vida, no sólo como simples herramientas, sino que modificando la mente y la forma de pensar de su "creador". En este contexto Ong explicita el valor que él atribuye al término media:

[...] el término "media" para indicar las nuevas modalidades tecnológicas a través de las cuales transformar la palabra, como la escritura, la imprenta y los dispositivos electrónicos. El término es útil [...] pero puede llevar al equívoco, llevándonos a pensar en la escritura, en la impresión 
y en los dispositivos electrónicos sólo como modos para "transportar la información”, en una especie de espacio intermedio entre una persona y otra. En realidad, cada uno de los así llamados "media" hace mucho más: hace posibles procesos mentales hasta ahora inconcebibles. Los "media" tienen más importancia dentro de la mente que fuera de ella. Con el uso de la escritura, de la imprenta o del ordenador, la mente no se convierte en una máquina que reduce su producción de pensamiento ("los conceptos") a un simple "dar forma", su memoria a un "almacenamiento de datos", sus recuerdos a una "recuperación de datos", su participación a la "circulación". Al contrario, la escritura, la imprenta y los ordenadores hacen posible que la mente pueda crear dentro de sí -y no sólo sobre la superficie inscrita o en los programas del ordenador- nuevas maneras de pensar, problemas anteriormente inconcebibles y nuevas maneras de crear respuestas.

La mente no entra en el alfabeto, en el libro impreso o en el ordenador tanto como el alfabeto, la imprenta o el ordenador entran en la mente, produciendo en ella nuevos estados de conciencia. (Ong, 1989: 52)

Pero tal vez el rasgo más interesante y original en este viaje a lo largo de la historia que Ong recorre es el hecho de que el viaje no es sólo de ida, sino también de vuelta; de hecho son continuas las interferencias que se pueden captar en los distintos momentos de transición. Baste con pensar el lugar de importancia que la oralidad ha vuelto a adquirir en nuestro tiempo, en el difuso emerger de formas de comunicación directa y en los muchos rasgos que hoy en día se encuentran en común con las antiguas culturas (los lemas publicitarios, por ejemplo, privilegian la parataxis, la redundancia, referencia evidente a una cultura oral), tanto que se puede hablar de una oralidad de retorno u "oralidad secundaria". "Sólo ahora, en la era electrónica, nos damos cuenta de las diferencias que existen entre oralidad y escritura; la era electrónica (cuya existencia depende de la escritura y de la imprenta) es también una era de 'oralidad de retorno', aquella del teléfono, de la radio, de la televisión...” (Ong, 1986: 21).

Y este constante saltar con naturalidad entre pasado y presente ("el futuro es algo del pasado" afirmaba McLuhan) hace fascinante la obra de Ong, y hace de ella el punto de partida para leer de manera diacrónica las etapas que caracterizaron la historia de la lectura.

Es útil acercarse a la oralidad y a la escritura de manera diacrónica, comparando las culturas orales y las quirográficas (basadas en la escritura) que coexisten en un dado periodo de tiempo. Pero es igualmente esencial la aproximación diacrónica o histórica [...] Homero y la televisión pueden iluminarse recíprocamente. (Ong, 1986: 20) 


\section{LOS MEDIA COMO METÁFORAS ACTIVAS: EL “MEDIUM ES EL MENSAJE”}

Otra aportación importante para la lectura de los pasos de transición ha sido el encuentro con la obra de aquél que el mismo Ong define como su más acreditado predecesor: Marshall McLuhan. Figura extremadamente poliédrica, considerado como el fundador de la Escuela de Toronto, McLuhan estuvo entre los primeros en comprender el impacto que los medios tenían y tendrían en la sociedad; su interés por los procesos cognitivos y la gran implicación de los medios de comunicación son los rasgos dominantes de su pensamiento, que culmina afirmando que las sociedades siempre han sido plasmadas más por la naturaleza de los medios a través de los cuales los hombres comunican que por el contenido de la comunicación.

Es una de las afirmaciones tal vez más controvertidas del "abad de los medios", que si por una parte justifica la mirada a veces perpleja de la comunidad científica y la acusación de una excesiva visión determinista, por otra parte es fundamental en la reconstrucción de una historia de la lectura que pone en el centro de su investigación precisamente al medium.

En una cultura como la nuestra, con una larga tradición de fraccionar y dividir para controlar, puede ser un choque que le recuerden a uno que, operativa y prácticamente, el medio es el mensaje. Esto significa simplemente que las consecuencias individuales y sociales de cualquier medio, es decir, de cualquiera de nuestras extensiones, resultan de la nueva escala que introduce en nuestros asuntos personales cualquier extensión (o tecnología nueva). (McLuhan, 1967: 15)

Los medios considerados como extensiones del hombre, como prolongaciones de sus sentidos (el teléfono amplifica en el espacio el oído y la voz al igual que la escritura extiende la memoria), como un sistema de interacciones bioquímicas en búsqueda constante de un equilibrio cada vez que se presente una nueva extensión.

Cada medium amplifica entonces las potencialidades sensoriales, físicas, intelectuales y cognitivas del individuo y esto causa una inevitable redefinición de las estructuras de la sociedad.

Partiendo de este principio McLuhan individúa dos categorías de medios: "medios calientes" y "medios fríos". Un medium es caliente cuando extiende un único sentido hasta una alta definición, o sea, hasta el estado en el cual los individuos están abundantemente colmados de datos.

Los medios calientes son a menudo, pero no siempre, los medios de la visión, y en este sentido la lectura está considerada como uno de ellos, ya que 
pide al lector mucha atención, intensificando el esfuerzo perceptivo de cada uno de sus órganos sensoriales; este modo no deja espacio para colmar o completar y conlleva, por lo tanto, una participación limitada.

Al contrario, un medium se define frío cuando conlleva una baja definición, es decir, cuando provee una cantidad limitada de información. Implica por tanto un elevado grado de cumplimiento por parte de la audience, pidiéndole una fuerte participación para que sea facilitada la percepción de modelos abstractos y la comprensión simultánea de las partes de la transmisión.

A menudo los medios fríos atañen al sentido del oído y entre los ejemplos preferidos por McLuhan están los así llamados encuentros seminariales, la televisión y los cómics. "Un medium caliente permite menos participación que un medium frío; una conferencia menos que un seminario, un libro menos que un diálogo" (McLuhan, 1967:31).

Para sintetizar la lectura que hace McLuhan de la dinámica de la comunicación entre medios y audience podríamos decir que cuanto más baja sea la definición de los mensajes del medio más alta será la participación, puesto que el receptor se verá obligado a seguir la información incierta. Los hot media, siendo más definidos, provocan una mayor participación en términos de implicación de masas, pero también una menor distancia subjetiva, una menor atención crítica y un menor empeño en la descodificación del mensaje por parte del usuario; por otra parte, los cool media, a causa de una menor definición, inducen a una menor masificación de la audience, promoviendo más atención subjetiva y más criticidad personal. En síntesis, aquéllos masifican mientras que éstos individualizan.

Esta distinción es especialmente útil en el camino que estamos a punto de emprender, puesto que nos permite comprender la dificultad, o mejor dicho, la imposibilidad de clasificar la lectura dentro de una de las dos categorías. Pensemos en las primeras prácticas de lectura, en la estrecha conexión con las formas de oralidad, en las obras contadas, cantadas o escuchadas por un público partícipe. Y también en las nuevas herramientas de lectura, en el libro electrónico por ejemplo, que implica al mismo tiempo más sentidos del lector y lo sitúa en una nueva dimensión de interacción con el medium.

Además, en la misma obra de McLuhan no se lee una dicotomía real entre caliente y frío, sino un cotinuum: de hecho, la incidencia de la distinción es relevante no sólo conforme al nivel de aculturación del usuario sino, sobre todo, respecto a la estrecha relación que hay entre el entorno, el contexto histórico y el medium. La fluidez que caracteriza el límite de tal distinción es, por tanto, la misma que volvemos a encontrar en la definición de los confines entre las etapas de una historia de la lectura en la cual el cambio, el "tránsito", es un rasgo imprescindible. 
El "medio es el mensaje" y "medios calientes, medios fríos" están entre las afirmaciones más controvertidas, debatidas y sin duda más significativas para comprender no sólo el estilo intencionalmente provocativo de McLuhan sino, sobre todo, la carga innovadora de su pensamiento, que culmina en una de las expresiones más conocidas acuñadas por el massmediólogo canadiense, la de "aldea global", que -dicho sea de paso- es el título de una de sus obras más relevantes.

"Aldea global" es en sí un oxímoron, el más "afortunado oxímoron" inventado hacia los años 60 por Marshall McLuhan, afirman los críticos, para describir la situación contradictoria en la que vivimos. Los dos términos del enunciado se contradicen mutuamente: "aldea" expresa algo pequeño, limitado, mientras que "global" significa el planeta entero.

Fuertemente enraizado y atento a las dinámicas y a las evoluciones de su tiempo y a la vez proyectado en leer los posibles escenarios futuros, McLuhan analiza cómo la cultura de la imprenta, claramente individualista y visual, se encuentra expuesta al rápido ocaso a raíz del avance de la interdependencia electrónica provocada por los medios electrónicos. Una nueva época eléctrica está sustituyendo a la anterior época mecánica, trazando un esmerado retrato de un hombre nuevo, un habitante de la aldea global, todavía en suspenso entre dos tecnologías, dos modos distintos de actuar y pensar.

El género humano será llevado a pasar del individualismo y la fragmentación a la adquisición de una identidad colectiva y en este paso deberá ponerse a la búsqueda de sus valores, de su integridad con un retorno al pasado (caracterizado por una base tribal, por formas sociales pre-industriales fundadas esencialmente en la oralidad) para después juntarse con el futuro. Un hombre, aquél descrito por McLuhan, que pretende comprender hasta lo más profundo la propia índole, consciente de sus acciones, pero al mismo tiempo en búsqueda de la claridad en el caos de la información, de los intercambios intensos de noticias propios del proceso de globalización, que pasa precisamente a través de las espesas redes telemáticas que envuelven el globo y está firmemente sustentada por la rápida difusión de las tecnologías de la comunicación.

Tras haber estallado durante tres mil años con medios tecnológicos fragmentarios y puramente mecánicos, el mundo occidental ha entrado ahora en una fase de implosión. [...] Estamos acercándonos rápidamente a la fase final de la extensión del hombre: aquélla en la que a través de la simulación tecnológica, el proceso creativo de conocimiento se extenderá colectivamente a la sociedad humana entera, al igual que, mediante los diferentes media, hemos extendido nuestros sentidos y nuestros nervios.

[...] En la era de la mecánica, hoy en decadencia, muchas acciones podían ser recibidas sin demasiadas preocupaciones. La lentitud de cada movimiento 
en general garantizaba que las reacciones seguirían con un retraso considerable. Hoy en día, en cambio, acción y reacción son casi contemporáneas. No vivimos, por así decirlo, mítica e integralmente, sino que seguimos pensando según los antiguos y fragmentados módulos de espacio y tiempo de la era pre-eléctrica.

El hombre occidental había derivado de la tecnología de la alfabetización la capacidad de actuar sin reaccionar. [...] Pero este desapego era un signo de no participación.

La aspiración de nuestra época a la totalidad, a la empatía y la consciencia en profundidad es un complemento natural de la tecnología eléctrica. La época de la industria mecánica que nos ha precedido ha encontrado su natural modo de expresión en la vehemente aserción de opiniones personales. Cada cultura y cada época tienen un modelo preferido de percepción y de conocimiento y tienden a prescribirlo para todos y para todo. [...] Profundamente persuadido de que sea posible llegar a comprender estas formas para utilizarlas de manera ordenada... (McLuhan, 1967: 9-12)

En este pasaje emerge uno de los componentes esenciales del pensamiento de McLuhan: el sentido de interrelación entre pasado, presente y futuro (como ya se ha dicho, retomado por el discípulo Ong), que se identifica con las tres eras de la historia humana. La primera, la tribal, caracterizada por el oído, se funda en la cultura oral. El hombre tribal, no alfabetizado, vive en un estado de intensa acentuación de la organización auditiva de toda la experiencia: la palabra posee una fuerza viva, un poder casi mágico. Un espíritu científico y analítico califica, en cambio, la era mecánica que, con la invención de la imprenta, amplifica la extensión de la vista. La palabra se convierte en un simple significado mental a menudo ausente de un destinatario claramente identificado. Se asiste a un proceso de destribalización, en el cual desaparece aquel sentido de fuerte adhesión a la sociedad que había caracterizado la primera era. La era mecánica, como se ha puesto en evidencia, ya ha cedido el paso a la era eléctrica en la que se asiste a la extensión de todos los sentidos, en una sinestesia absoluta. La tecnología eléctrica, no especialista como la mecánica, lleva al género humano a reactivar aquellas dinámicas sociales que habían caracterizado la era tribal (re-tribalización). El hombre vuelve a asomarse más allá del espacio geométrico organizado por las sociedades alfabetizadas y más aún por la mentalidad tipográfica. Del oído a la vista, y luego otra vez de la vista al oído, una vuelta a la oralidad que caracteriza a la era eléctrica.

Es éste el camino que estamos recorriendo. La última frontera está representada por aquellas tecnologías que nos permiten el acceso directo a bases de 
datos, o bien el diálogo a distancia entre las personas [...]

La alfabetización empujó al hombre fuera de la tribu, le dio el ojo en lugar de la oreja y sustituyó su sentimiento de adhesión colectiva absoluta y en profundidad por valores lineales y visivos y por una conciencia fragmentaria e individualista.

De ahí una posible nota de optimismo: si la civilización electrónica ensalzare de nuevo el oído, el sentido de la proximidad, tendremos la esperanza de que las divisiones del mundo se puedan atenuar. (McLuhan, 1982: 43-46)

Sin detenernos en esta última "nota de optimismo" del religioso massmediólogo, que merecería una reflexión sobre el grave y peligroso asunto de la distancia que existe entre quien tiene acceso efectivo a las nuevas tecnologías de la comunicación y la información y quien, por razones casi siempre ligadas a condiciones socioeconómicas inferiores, se encuentra terminantemente excluído de ellas (digital divide), es necesario prestar atención a la clave que McLuhan, en el genial intento de encontrar una conexión entre pasado, presente y futuro, utiliza para leer la historia de las sociedades, o más bien, de los media y el poder que éstos ejercen sobre el hombre, al menos hasta cuando, saliendo de la condición de "servomecanismo" ("relación robótica con sus mismos inventos" lo define McLuhan), no vuelva a apoderarse de su extensión autoredefiniéndose a raíz de los nuevos cambios.

El medium se convierte en una fuerza desconocida para quien lo utiliza. Por esta razón todas las sociedades se encuentran inicialmente aturdidas por el uso de nuevas tecnologías. Ninguna cultura, en ningún período histórico, se ha dado cuenta perfectamente, ni siquiera a posteriori, de los efectos producidos por sus medios utilizados como expresiones de ellos mismos en un sistema global. (McLuhan, 1989: 125)

McLuhan, en el texto escrito con Quentin Fiore, llega incluso a decir, con un hábil juego de palabras, que "el medio es el mensaje":

Todos los medios nos arrollan por completo. Son tan penetrantes en sus consecuencias personales, políticas, económicas, estéticas, psicológicas, morales, éticas y sociales, que no dejan ninguna parte de nosotros intacta, virgen, invariada. El medio es el mensaje. Cada interpretación de la transformación social y cultural es imposible sin un conocimiento del modo en que los medios funcionan como entornos. (McLuhan y Fiore, 1968: 26)

El medium, extensión de alguna facultad humana psíquica o física, parece "masajear" al usuario; si por una parte lo tranquiliza, reconforta y confirma, al mismo tiempo lo condiciona hasta el punto de plasmar su mente. 
Junto con las obras del intelectual canadiense hasta aquí mencionadas, fundamental en la reconstruccción de una historia de la lectura es sin duda The Gutenberg Galaxy. The making of the Typographic Man (La galaxia Gutenberg. Génesis del homo typographicus), escrita en 1961 y publicada al año siguiente por la Toronto University Press. En esta obra McLuhan concentra su reflexión sobre la invención, o mejor dicho, sobre la afirmación de la imprenta de carácteres móviles en el mundo occidental.

Tras subrayar el papel prioritario que las tecnologías y los medios siempre han tenido en las sociedades y como éstas pueden ser consideradas plenamente como factores determinantes en los procesos de transformación social, vuelve a recorrer las etapas de la historia humana con una técnica que él mismo define a modo de mosaico, funcional a la reconstrucción de un recorrido casi nunca lineal, hecho de cambios, interconexiones, equilibrios y desestabilizaciones que han caracterizado los diferentes periodos históricos.

La Galaxia Gutenberg aborda el mismo tema con un método a modo de mosaico o de campo. Esta imagen compuesta de numerosos datos y citas relevantes constituye la única herramienta práctica para evidenciar en la historia las operaciones casuales.

[... Tal vez hubiera sido mejor utilizar en vez de "galaxia" la palabra "entorno". Cada tecnología tiende a crear un nuevo entorno humano. La escritura y el papiro crearon el entorno social que tenemos en mente cuando hablamos de los imperios de la antigüedad. El estribo y la rueda crearon entornos totalmente nuevos de una envergadura inmensa. Un entorno tecnológico no es solamente un contenedor pasivo de hombres, sino un proceso activo que remodela los hombres al igual que las demás tecnologías. En nuestra época el repentino paso de la tecnología mecánica de la rueda a la tecnología de los circuitos eléctricos constituye uno de los principales cambios de toda la historia. La imprenta de carácteres creó un entorno nuevo y totalmente inesperado: el PÚBLICO. (McLuhan, 1976: 20)

Este es un pasaje fundamental para un recorrido que ve al lector como protagonista y que reconoce en la invención de la imprenta de carácteres móviles una de las revoluciones que más han contribuido a forjar la experiencia humana y uno de los pasajes fundamentales para leer la historia de la cultura occidental.

El lector es para nosotros el "público" del cual habla McLuhan, es el individuo arrollado por las transformaciones tecnológicas en las esferas más íntimas de su equilibrio psico-perceptivo y en las relaciones con la comunidad a la que pertenece. Las civilizaciones percibidas, por lo tanto, como espejo del 
sistema sensorial individual y colectivo, y la imprenta leída como tecnología que ha ensalzado el sentido de la vista y que ha lanzado su radio de acción sobre un público más vasto que nunca.

La tecnología del manuscrito no tenía ni la intensidad ni la capacidad de extensión necesaria para crear un público a escala nacional. Las que en los últimos siglos hemos llamado "naciones" no precedieron (ni hubieran podido) el advenimiento de la tecnología de Gutenberg más de lo que no podrán sobrevivir al advenimiento de los circuitos eléctricos con su capacidad de implicar totalmente a todos los hombres entre sí.

El carácter peculiar del "público" creado por la palabra impresa fue el de una conciencia intensa y visivamente orientada, tanto en el individuo como en el grupo. Las consecuencias de este intenso estrés visivo, con el creciente aislamiento de la vista con respecto a los demás sentidos están ilustradas en este volúmen. Su tema principal y la extensión de las modalidades visivas de continuidad, uniformidad e interconexión en la organización tanto del tiempo como del espacio. Los circuitos eléctricos no permiten la extensión de las modalidades visivas sino en medida muy inferior a la capacidad visiva de la palabra impresa. (McLuhan, 1976: 20)

Esta estrecha conexión entre tecnología y esfera sensorial, entre hombre y medio, lleva a McLuhan a definir la imprenta como un verdadero "recurso natural", y por consiguiente al libro impreso como una mercancía, "la primera mercancía uniforme y repetible".

La tipografía no es solamente una tecnología, es también un recurso natural, un producto básico, como el cartón, la madera o la radio; y como cada materia prima, ella condiciona no solamente las relaciones entre los sentidos del individuo sino también los modelos de interdependencia comunitaria. [...] La uniformidad y la repetibilidad no sólo creó los mercados modernos y el sistema de precios, estrechamente relacionados con la alfabetización y el desarrollo de la industria. [...] La imprenta en sí era un bien de consumo, un nuevo recurso natural que nos enseñó también como aprovechar cualquier otro tipo de recurso, nosotros mismos incluídos. (McLuhan, 1976: 222-223)

Un último aspecto, pero no por ello menos importante, que hace de la obra de McLuhan uno de los principales puntos de partida de mi trabajo, es el método utilizado por él en el análisis de la historia del hombre a través de los componentes culturales y massmediáticos de la civilización, método que él mismo define, como ya se ha dicho, a modo de "mosaico". La escritura discontinua, llena de ejemplos, citas, referencias no siempre fácilmente 
descifrables, el saltar casi "esquizofrénico" entre los diferentes ámbitos disciplinarios, sus boutades provocativas, la aparente falta de una "lógica" clara hacen del "método McLuhan" una herramienta interesante para analizar un momento de tránsito, como es el paso a la era digital que estamos viviendo hoy en día, que todavía no se puede encerrar en esquemas precisos y no se puede observar desde un "punto de vista fijo" que permita definir con rigor el objeto de investigación.

El que McLuhan adopta en su investigación es un "punto de vista móvil" (Iser), como evidencia Elena Lamberti:

El "punto de vista móvil" [...] es una estrategia expositiva que obliga al lector a entrar inmediatamente en juego y a convertirse en co-actor del mismo proceso de observación. Lo que se observa ya no es un lienzo otro y distante. Es el mundo en su totalidad, una obra de arte compleja que envuelve, implica y obliga a reaccionar rápidamente, transformando al mismo observador en "punto de fuga" de la observación. (Lamberti, 2000: 11)

McLuhan observa la realidad no de frente, sino con una mirada transversal, capaz de captar matices y ángulos diferentes; mirar los sucesos desde un punto de vista móvil permite analizar momentos no definidos, fluidos, como son los de "tránsito".

\section{BIAS Y BRAINFRAMES}

Junto a la que es la figura central, McLuhan, se añaden Harold Innis y Derrick de Kerckhove, respetivamente maestro y dicípulo de McLuhan, y también otros intelectuales, estudiosos de diferentes perspectivas de la historia de la comunicación, como Jack Goody y Eric Havelock, cuyas contribuciones se examinarán en este texto.

Innis muestra cómo los procesos sociales, culturales, políticos y económicos intrínsecos en las civilizaciones que se han subseguido a lo largo de la historia están caracterizados y fuertemente condicionados por la comunicación y por las tecnologías que a ella se aplican.

Su formación de economista e historiador, principalmente interesado por el análisis de los procesos de producción de los recursos primarios y sobre todo por la influencia de estos últimos en la evolución y el desarrollo de la sociedad, hace de Innis un observador original de la historia de los media. Su aproximación innovadora se percibe desde sus primeras reflexiones, en las que extiende el concepto de monopolio, adoptado esencialmente por una dimensión de tipo económico ligada a los medios de producción, a los medios 
de comunicación. El monopolio ejercido por los media, la posesión y el control de la información, son para Innis las principales herramientas para ejercer el poder.

El Tratado de Versalles reconoció el impacto de la imprenta aceptando el principio de los derechos de la autodeterminación y destruyó grandes organizaciones políticas como el imperio austríaco. La comunicación, basada en la vista en términos de imprenta y fotografía, había desarrollado un monopolio que amenazaba con destruir la civilización occidental antes en guerra y después en paz. Este monopolio ensalzaba el individualismo y creaba la ilusión con lemas como democracia, libertad de imprenta y libertad de palabra.

El efecto desastroso del monopolio de la comunicación basado en la vista aceleró el desarrollo de un competititvo tipo de comunicación basado en el oído, con la radio y con la combinación del sonido con el cine y la televisión. Lo impreso fue superado en eficiencia por la radiodifusión y el altavoz. Los jefes políticos fueron capaces de apelar directamente a los votantes y construir la presión de la opinión pública sobre la asemblea legislativa.

[...] En Europa la apelación al oído hizo posible la destrucción de los resultados del Tratado de Versalles como había sido registrado en el mapa geográfico político fundado en la autodeterminación. La subida al poder de Hitler fue facilitada por el uso del altavoz y de la radio. A través de la lengua hablada él podía apelar a las naciones y a los grupos minoritarios. Los alemanes en Checoslovaquia podían ser alcanzados por la radio igual que los alemanes en Austria. Las fronteras políticas propias de la industria de la imprenta desaparecieron con el nuevo medio de comunicación. La lengua hablada ofreció una nueva base a la explotación del nacionalismo y un recurso mucho más eficaz para apelar a un número mayor de individuos. El analfabetismo ya no era una barrera importante. (Innis, 1982: 103)

En este fragmento, en el cual resulta inmediatamente evidente la originalidad del autor en la relectura de un periodo específico histórico, Innis demuestra cómo los medios de comunicación que necesariamente interactúan con el público, si bien por un lado dejan libertad de crítica a la audience, por el otro se exponen al riesgo de ser herramientas de manipulación de las conciencias por parte del poder.

La reflexión de Innis parte, por tanto, precisamente del considerar, por un lado, la centralidad del medio y de la comunicación del conocimiento como bases de la relación social y económica entre los hombres, y por otro lado de subrayar cómo la capacidad de los medios, especialmente de la escritura y de la producción de un texto escrito, llega a configurarse como una herramienta de poder para afirmar un "monopolio del saber". 
Los monopolios del conocimiento se han desarrollado y han decaído, en parte, por efecto del medio de comunicación sobre el cual se habían construido, y su historia está hecha de una alternancia entre los medios que insistían en la religión, en la descentralización y en el tiempo, y los medios que en cambio insistían en la fuerza, en la centralización y en el espacio. (Innis, 2001: 253)

Es en este contexto donde Innis introduce el concepto de "medio dominante" que se impone en una civilización y cuando pierde su supremacía desestabiliza la sociedad hasta que un nuevo medio no conquiste un rol de supremacía y se convierta en característico de la civilización sucesiva. Esta continua tendencia de la tecnología hacia nuevas organizaciones de las formas de comunicación del saber y la del hombre de adaptarse a estas nuevas formas (a los nuevos medios) y el intento incesante de dominarlas y someterlas a sus propios intereses, la define Innis como bias.

Precisamente en función de esta "tendencia" interna, bias (que a veces puede parecer influencia, vínculo o incluso prejuicio), Innis subdivide los media en relación a la dimensión que más expresan: algunos, como el papiro, la arcilla y la piedra se inclinan hacia el tiempo; otros, como el papel y el pergamino, hacia el espacio. Los medios de comunicación modernos como la radio y la televisión, en cambio, han determinado una progresiva erosión de la importancia del tiempo como duración histórica (todavía presente en la prensa, que hacía hincapié en la duración de la información que transmitía) en favor de un achatamiento sobre el instante. Y justo en función de las coordenadas de tiempo y espacio que los media amplifican o no, y del equilibrio que una sociedad consigue crear entre estos dos parámetros, se determina, según Innis, la vida y el éxito de la sociedad misma ("imperio").

La organización política a gran escala conlleva la solución de los problemas de espacio, en términos de eficiencia administrativa, y de los problemas de tiempo, en términos de continuidad. La elasticidad de la estructura requiere una búsqueda continua de habilidades y competencias, y hace necesarios ataques persistentes al monopolio del conocimiento. Es posible alcanzar la estabilidad sólo con una exacta consideración de los que son los límites de las herramientas de gobierno, y no solamente de sus potencialidades.

La concentración en un medio de comunicación lleva consigo un condicionamiento en el desarrollo cultural de la civilización, que en consecuencia estará interesada en la importancia del espacio y, por consiguiente, en la organización política o del tiempo, y por lo tanto, en la organización religiosa. La introducción de un segundo medio tiende a frenar la influencia del primero y a crear las condiciones adecuadas para el crecimiento del imperio. El imperio bizantino, por ejemplo, emergió de una fusión entre los efectos del papiro sobre la organización política y los efectos del pergamino sobre 
la organización eclesiástica. El dominio del pergamino llevó, en la historia de Occidente, al monopolio de la organización eclesiástica, que a su vez provocó la introducción del papel, favorable por otra parte al desarrollo de las instituciones políticas. Con la invención de la imprenta, el papel facilitó el desarrollo efectivo de las vernáculas, expresando su vitalidad en el aumento del nacionalismo. La adaptabilidad del alfabeto a la producción industrial a gran escala se convirtió en la base de la alfabetización, del aumento de la publicidad y del comercio. El libro, como producto especializado de la imprenta, y sucesivamente el periódico, reforzaron la posición de la lengua como base del nacionalismo.

En Estados Unidos, la hegemonía de la prensa cotidiana conllevó el crecimiento de monopolios de comunicación a gran escala, fundamentados en la gestión del espacio, con la consiguiente subestimación de los problemas relacionados con el tiempo; y el monopolio de las asociaciones de la prensa, en este sentido, reforzaba el monopolio ejercido por los periódicos metropolitanos en el ámbito regional y local.

El monopolio del conocimiento fundado en la dimensión del espacio, sin embargo, fue obstaculizado por el nuevo medio: la radio; la cual llevó consigo una nueva preocupación por los problemas del tiempo, visible en las políticas de programación y planificación estatal. La dependencia de los periódicos, en Estados Unidos, implicaba una condición de inestabilidad, que en todo el mundo occidental ha facilitado el uso de la fuerza como factor estabilizador. La capacidad de desarrollar un sistema de gobierno en el cual la influencia de la comunicación pueda ser controlada, y en el cual sea posible alcanzar una gestión equilibrada del espacio y del tiempo, permanece como el problema del Imperio y de todo el mundo occidental. (Innis, 2001: 254)

El análisis de Innis se convierte entonces en una herramienta de gran interés para leer los momentos de tránsito pero sobre todo para comprender los cambios actuales. Si pensamos en los nuevos medios de comunicación, como la computadora y los medios digitales en general, notamos como éstos, respecto al pasado, no son "exclusivos" en relación a las dimensiones de espacio y tiempo: de hecho utilizan como base para comunicar un código de tipo híbrido, situado en la intersección de las dos coordenadas. Las diferentes maneras en las que tal forma de comunicación se manifiesta pueden, además, inclinarse más hacia el tiempo (pensemos en los metadatos, en los archivos) o hacia el espacio (correo electrónico, chat), dejando abiertas nuevas posibilidades interpretativas también respecto al concepto de bias localizable, pero no definitivamente enmarcable, en las actuales tecnologías informáticas. El interés por la tendencia de los media que caracterizó civilizaciones pasadas o diversas de aquélla en la que vivimos nos permite, afirma Innis, comprender nuestra sociedad y la manera en la que los medios de comunicación influyen en ella. 
En cuanto nos aventuramos en esta investigación estamos obligados a conocer la tendencia del período en el que actuamos. El interés por la tendencia de otras civilizaciones puede en sí mismo sugerir una tendencia de la nuestra.

Nuestro conocimiento de otras civilizaciones depende en gran medida de las características de los medios de comunicación utilizados por cada civilización por sus características de conservación o bien por haberse vuelto accesible a través de los descubrimientos [...]

La tendencia de la civilización moderna relativa al periódico y a la radio hará suponer una perspectiva en consideración de civilizaciones dominadas por los medios de comunicación. No podemos hacer otra cosa que estar alerta sobre las consecuencias de esta tendencia y tal vez esperar que la observación de las consecuencias de otros medios de comunicación sobre otras civilizaciones nos permita ver más claramente la tendencia de la nuestra. (Innis, 1982: 54)

De Kerckhove, apoyando y complementando las tesis expresadas hasta aquí, en su obra Brainframes. Mente, tecnologia, mercato (Brainframes. Mente, tecnología, mercado), demuestra como los brainframes, o los modelos mentales estimulados por los nuevos medios, no están plasmados por el contenido que conllevan los medios sino por sus características estructurales, y analiza la estrecha conexión entre tecnología y psicología estudiando el impacto y la influencia de la primera sobre el desarrollo de la segunda.

Un brainframe es algo distinto a una actitud, una mentalidad, aunque sea todo esto y mucho más. Si bien aprovecha y filtra nuestra visión del mundo, éste no es exactamente un par de gafas de tipo particular - puesto que un brainframe nunca está localizado en la estructura superficial de la conciencia, sino en su estructura profunda. (De Kerckhove, 1993: 11)

Cada tecnología nos modifica entonces de manera profunda: fisiológicamente, alterando la organización neuronal; psicológicamente, alterando las categorías mentales a través de las cuales interpretamos el mundo. Un concepto clave de toda la argumentación es la idea de que el brainfame, o marco cerebral, es la manera en la que las tecnologías enmarcan el cerebro humano en una estructura. El frame es un esquema de representación, pero ampliando su sentido se convierte en un marco o una estructura que encuadra las capacidades de expresión.

De Kerckhove resalta la centralidad de la escritura en la creación de un braiframe, en el sentido de una estructuración debida a la interacción entre un sistema biológico con características morfológicas y funcionales dadas (diferencias entre los dos hemisferios) pero profundamente plásticas, y un entorno mutable. Como demostración de ello el intelectual belga cita el 
ejemplo del acto de escritura que ocurre, al menos en la mayoría de los casos, de izquierda hacia derecha: lo cual parece derivar del hecho de que el hemisferio izquierdo, predispuesto al análisis secuencial de los datos y a la percepción del campo visivo derecho, induce el cerebro a desplazar la atención secuencial hacia el lado derecho. La técnica de la escritura, en síntesis, envía información a la mente creando un modelo estructural de la organización de los datos sensoriales; la interacción conlleva entonces una transformación.

Para De Kerchkove, a un nivel mental profundo, el brainframe de la alfabetización influye en el modo en el que organizamos nuestros pensamientos, creando así "marcos mentales". A tales marcos internos se añaden también marcos externos, como las pantallas de la televisión o de la computadora, que el autor define como frames de frames, es decir marcos de marcos. Esto hace de la televisión y de la computadora herramientas todavía más fuertes y potentes de la escritura, puesto que a través de un bastidor externo llegan a modificarnos internamente. La pantalla de la computadora, por ejemplo, se presenta con mapas y ventanas interactivas que permiten ampliar las mismas posibilidades de uso por parte del usuario.

Los nuevos media, según De Kerckhove, proponen un nuevo tipo de comunicación en el que hay un intercambio entre el mundo exterior y nuestro yo más profundo, más intenso de lo que nunca ha occurrido en el pasado. La teoría de De Kerckhove según la cual cada nuevo medio de comunicación configuraría nuestros hemisferios cerebrales delineando sustanciales modificaciones fisiológicas y cognitivas nos permitirá analizar los cambios actuales y la afirmación de nuevos media desde una perspectiva ulterior.

\section{Conclusiones}

La definición de "lector híbrido", centro de nuestra investigación, que al parecer proporcionaba una sombra de incertidumbre sobre nuestro futuro y la transición que estamos viviendo, como hemos demostrado con la ayuda dada por la Escuela de Toronto, indica una condición que siempre ha caracterizado la vida del lector que en el cambio, e incluso en la incertidumbre, ha crecido y evolucionado adquiriendo nuevas habilidades y conocimientos.

La propia lectura es, a fin de cuentas, una competencia que el hombre ha hecho suya a través de un proceso complejo, no siendo un acto connatural al individuo y a sus naturales potencialidades.

Cuando se lee, de hecho, hay múltiples representaciones mentales que interfieren entre ellas y, además, a diferentes niveles. Esto da el disparo de 
salida a una amplia gama de estrategias e implica la articulación de diversas operaciones metacognitivas, haciendo de la lectura una de las conquistas más costosas pero también más preciadas para la humanidad, porque ha desencadenado en ella una infinita serie de cambios.

Lo anteriormente dicho nos permite ampliar el término bíbrido para todos aquellos lectores que, con la llegada de una nueva tecnología, han tenido que adaptarse al cambio, adquiriendo nuevas estrategias para responder a la evolución en curso, sin perder todavía la conciencia del pasado y, sobre todo, el legado que éste dejó.

El análisis que hemos explorado nos ha demostrado que, con el surgimiento de una nueva tecnología y de nuevos medios de comunicación, las prácticas y formas de lectura no cambian tan bruscamente: el lector efectúa un recorrido para aprovechar las innovaciones que encuentra y elabora el significado funcional de sus necesidades. Esta mediación continua, o mejor aún, remediación que el lector cumple, es la que Cavallo y Chartier llaman "dialéctica de la coerción y de la invención":

Los textos siempre se dan a conocer a sus lectores en formas (manuscritas o impresas, escritas u orales) que los hacen sujetos a ciertas restricciones, sin destruir su libertad. [...] Los lectores toman posesión de los libros (u otros objetos impresos), les dan un sentido, les proporcionan sus expectativas. (Storia della lettura 1998: XLI-XLII)

El lector híbrido es el mediador de estos pasos (que a menudo hemos llamado, quizá incorrectamente, revoluciones) y es en su capacidad de "notar" el cambio y aprovecharse del poder innovador que éste conlleva donde se encuentra el éxito de un nuevo medium y, al mismo tiempo, la co-existencia de formas del pasado. "La Musa aprendió a leer y escribir sin dejar de cantar" (Havelock, 2005: 29).

Aún a sabiendas de no poder entender plenamente el alcance de una "revolución" que todavía está teniendo lugar, nos damos cuenta de que, pese a todo, a la luz del recorrido que hemos concluido no podemos compartir la mayoría de las perplejidades sobre el futuro del libro, de la biblioteca y de la lectura, que todavía son objeto de debate entre los estudiosos de diferentes disciplinas. 
En cuanto al libro impreso, leyendo después de los pasajes que hemos analizado una cita provocativa de Umberto Eco, "El libro es como la cuchara, el martillo, la rueda, las tijeras, una vez que ya están inventados, no se puede hacer mejor" (Carrière y Eco, 2009: 16), podemos afirmar que el libro, como la cuchara que con el tiempo ha cambiado de aspecto y ha sido realizada con diferentes materiales, pero siempre ha preservado su utilidad manteniendo esa función para la cual había sido creada, conserva y siempre conservará un papel principal como apoyo a la cultura y como mediador del saber y el conocimiento.

Por lo que respecta a la lectura, habiendo demostrado que existe una correlación positiva (a pesar de los tópicos) entre la lectura y el uso de una variedad de medios (Solimine, 2010), nos parecen atractivas y, cuanto menos, tranquilizadoras las reflexiones de Luca Ferrieri:

La lectura encuentra alimento y no competencia en las diabluras de la comunicación electrónica (ventaja de las informaciones bibliográfica en la red). La lectura, la más temporal de las artes, la única que por simpatía hacia una vida efímera acepta su mortalidad, que disfruta de su consumirse, será, por ello, la que enterrará a todas las demás. La mejor garantía contra la extinción de la lectura consiste en su placer. (La biblioteca ibrida, 2003: 88)

Cada paso trae consigo esperanzas y dudas, entusiasmos y malos humores; las mutaciones tecnológicas, las transformaciones de los lenguajes han cambiado los media pero no los han eliminado. Es el lector quien opta por esa libertad inherente a la lectura de la que habla Cavallo y Chartier; y por lo demás, como dice Nunberg, "cuando todo es posible, no se renuncia a nada" (Nunberg, 1996: 20).

\section{REFERENCIAS}

Barilli, Renato. 1970. "Walter Ong e le tre età della parola". Il Mulino 2 (Bologna, Società editrice il Mulino): 265-274.

Carrière, J. Claude y Umberto Eco. 2009. Non sperate di lberarvi dei libri. Milno: Bompiani.

De Kerckhove, Derrick. 1993. Bralnframes. Mente, tecnologia, mercato. Bologna: Baskerville. Ed. or. 1991. Brainframes: Mind Technology and Market. Utrecht: Bosch \& Keuning.

Havelock, Eric A. 2005. La Musa impara a scrivere. Riflessioni sull'oralità e l'alfabetismo dall'antichità al giorno d'oggi. Roma-Bari: Laterza. Ed. or. 1986. The Muse Learns to Write: Reflections on Orality and Literacy from Antiquity to the Present. New Haven and London: Yale University Press. 
Innis, Harold A. 1982. Le tendenze della comunicazione. Milano: SugarCo. Ed. or. 1951. The Bias of Communication. Toronto: University of Toronto Press.

Innis, Harold A. 2001. Impero e Comunicazioni. Roma: Meltemi. Ed. or. 1950. Empire of Comunications. Oxford: Oxford University Press.

Jäger, Ludwig. 2003. "La parola crea il mondo". Mente \& Cervello 5 (settembre-ottobre) (Roma, Gruppo Editoriale l'Espresso).

La biblioteca ibrida. Verso un servizio informativo integrato. 2003. A cura di Ornella Foglieni. Milano: Editrice Bibliografica.

Lamberti, Elena. 2000. Marshall McLuban. Milano: Bruno Mondadori.

McLuhan, Marshall. 1976. La galassia Gutenberg: nascita dell'uomo tipografico. Roma: Armando. Ed. or. 1962. The Gutenberg Galaxy. The Making of Typographic Man. Londra: Routledge \& Kegan Paul.

McLuhan, Marshall. 1967. Gli strumenti del comunicare. Milano: Il Saggiatore. Ed. or. 1964. Understanding Media: The Extensions of Man. New York: McGraw-Hill.

McLuhan, Marshall. 1982. Dall'occhio all'orecchio. Roma: Armando Editore.

McLuhan, Marshall. 1989. Il villaggio globale. XXI secolo: trasformazioni nella vita e nei media. Milano: SugarCo. Ed. or. 1989. The Global Village: Transformations in World Life and Media in the 21th Century. New-York: Oxford University Press.

McLuhan, Marshall y Quentin Fiore. 1968. Il medium è il massaggio. Milano: Feltrinelli. Ed. or. 1967. The Medium is the Massage. London: Penguin Books.

Nunberg, Geoffrey. 1996. The Future of the Book. Berkeley: University of California Press.

Ong, Walter J. 1970. La presenza della parola. Bologna: Il Mulino. Ed. or. 1967. The Presence of the Word: Some Prolegomena for Cultural and Religious History. New Haven:Yale University Press.

Ong, Walter J. 1971. Rethoric, Romance, and Technology. Ithaca and London: Cornell University Press.

Ong, Walter J. 1986. Oralità e scrittura: le tecnologie della parola. Bologna: Il Mulino. Ed. or. 1982. Orality and Literacy: The Technologizing of the Word. Londra- New York: Methuen.

Ong, Walter J. 1989. Interfacce della parola. Bologna: Il Mulino. Ed. or. 1977. Interfaces of the Word. Ithaca: Cornell University Press.

Solimine, Giovanni. 2010. L'Italia che legge. Roma-Bari: Laterza.

Storia della lettura nel mondo occidentale. 1998. A cura di Guglielmo Cavallo e Roger Chartier. Roma-Bari: Laterza.

Para citar este texto:

Ranfa, Elena. 2018. "La lectura entre sociología y massmediología”. Investigación Bibliotecológica: archivonomía, bibliotecología e información 32 (75): 83-108.

http://dx.doi.org/10.22201/iibi.24488321xe.2018.75.57948 